\title{
From Standard of Care to the Neoadjuvant Model as an Innovative Platform for Exploring Promising Combinations in Breast Cancer
}

\author{
Cristina Saura \\ Breast Cancer Unit, Medical Oncology Service, Vall d'Hebron University Hospital, Barcelona, Spain
}

By demonstrating similar distant recurrence-free survival and overall survival $[1,2]$ compared to adjuvant treatment, preoperative chemotherapy for patients diagnosed with breast cancer was established as a standard of care. Additional benefits also make neoadjuvant chemotherapy a very attractive option in clinical practice, including the feasibility of increased conservative surgery with better cosmetic outcomes, the ability to test tumor sensitivity to the treatment received in vivo with the intrinsic prognostic information on different subtypes [3], and the ability to treat micrometastases from the very outset.

Neoadjuvant endocrine therapy also plays a role in the management of postmenopausal hormone receptor(HR)-positive/HER2negative breast cancer because it can achieve similar response rates as chemotherapy but with significantly less toxicity. This suggests that this therapy should therefore be reconsidered as a possible option in the appropriate setting [4]. Moreover, since neoadjuvant therapy allows for the testing of novel drugs before surgery and facilitates preliminary data to support the further development of combinations, these qualities also prove its worth in breast cancer research. As is the case with the management of breast cancer in general, this disease cannot be understood in the neoadjuvant context if it is not separated into the three main subtypes that we recognize in clinical practice: HR-positive, HER2-positive, and triplenegative tumors.

For this issue of BREAST CARE with a focus on 'neoadjuvant treatment in breast cancer', we have selected three papers that bring us up to speed on the very latest research advances and current opportunities within this field [5-7].

The first of this trio tackles the surgical management of patients after treatment following the neoadjuvant strategy and different approaches to de-escalate breast and axilla surgery [5]. The increased use of neoadjuvant therapy in the treatment of early breast cancer has challenged our surgeons to not only finely tune their techniques towards less extensive surgery but also to take advan- tage of the downstaging of disease to reduce morbidity associated with surgical therapy of the breast and axilla.

Since neoadjuvant chemotherapy has been associated with more frequent local recurrence than adjuvant chemotherapy [2], delivering optimal locoregional treatment is critical. Strategies to mitigate this reality should be carefully considered. The main discussion in this part of the review focuses extensively on the multidisciplinary management of patients, accurate radiological assessment prior to and following treatment, the type of markers and tracers that should be used, and the need to precisely tailor the type of surgery to the response achieved by each and every patient [5].

The second contribution offers a comprehensive review covering what has been reported in the literature on neoadjuvant systemic therapy in early/locally advanced breast cancer [6]. Among others, two of the topics addressed are optimal patient selection for neoadjuvant therapy based on clinical and biological features and how best to assess response after chemotherapy (i.e. pathologic complete response, residual cancer burden) and endocrine therapy (i.e. PEPI score, Ki67 after 2 weeks). Over the last few years, different drugs beyond anthracyclines and taxanes have been evaluated. This article reviews the accumulated evidence in triple-negative breast cancer that either argues in favor of or against the addition of platins and antiangiogenics as well as the use of nab-paclitaxel. Regarding HER2-positive disease, results from the major trials supporting dual anti-HER2 blockade in clinical practice are also discussed in this paper. Finally, concerning luminal tumors, the authors look at the currently available chemotherapy options as well as the optimal type and duration of endocrine therapy.

The third paper of this collection focuses on the neoadjuvant model as an opportunity to assess novel biomarkers and new therapies towards a better guidance of drug development in the adjuvant or metastatic setting [7]. For years, the neoadjuvant model has been positively embraced as a platform to advance clinical research by answering relevant questions in breast cancer with less patients

\section{KARGER}

(c) 2018 S. Karger GmbH, Freiburg

Fax +497614520714
Cristina Saura, $\mathrm{MD}, \mathrm{PhD}$ 
involved and less time invested. As an approach, it can also provide extremely valuable insights that could be analyzed upon biopsies performed during treatment or during surgery after exposure to the particular drug under evaluation.

This contribution then provides an update on the most promising therapies evaluated or currently under study for each breast cancer subtype, accompanied by the clinical and biological endpoints addressed in each highlighted trial. Together with their strengths and limitations, different models within neoadjuvant studies are also described. These include the classical randomized phase II trials combining a new drug to the standard of care, as well as the presurgical window of opportunity trial design as a platform for testing novel anti-cancer therapies that involve a short course of treatment prior to surgery.

For some discussion points, Escrivá-de-Romaní et al. [7] come to some very clear conclusions, while for others, thought-provoking questions are raised that can and will only be answered by means of future research.

In conclusion, in this focus on neoadjuvant therapy in breast cancer, all three articles provide an excellent summary of 'what is hot' in the field in 2018.

\section{References}

Fisher B, Bryant J, Wolmark N, et al: Effect of preoperative chemotherapy on the outcome of women with operable breast cancer. J Clin Oncol 1998;16:2672-2685.

- Early Breast Cancer Trialists' Collaborative Group (EBCTCG): Long-term outcomes for neoadjuvant versus adjuvant chemotherapy in early breast cancer: meta-analysis of individual patient data from ten randomised trials. Lancet Oncol 2018;19:27-39.

3 Cortazar P, Zhang L, Untch M, et al: Pathological complete response and long-term clinical benefit in breast cancer: the CTNeoBC pooled analysis. Lancet 2014; 384:164-172.
Spring LM, Gupta A, Reynolds KL, et al: Neoadjuvant endocrine therapy for estrogen receptor-positive breast cancer: a systematic review and meta-analysis. JAMA Oncol 2016;2:1477-1486

5 Cordoba O, Carrillo-Guivernau L, Reyero-Fernández C: Surgical management of breast cancer treated with neoadjuvant therapy. Breast Care 2018;13:DOI: $10.1159 / 000491760$
6 Pernaut C, Lopez F, Ciruelos E: Standard neoadjuvant treatment in early/locally advanced breast cancer. Breast Care 2018;13:DOI: 10.1159/000491759.

7 Escrivá-de-Romaní S, Arumi M, Zamora E, Bellet M: Neoadjuvant model as a platform for research in breast cancer and novel targets under development in this field. Breast Care 2018;13:DOI: 10.1159/000492122. 